\title{
The use of methylphenidate for physical and psychological symptoms in cancer patients: a review
}

\begin{abstract}
Background: One of the goals of cancer treatment is symptoms management especially at the end stage. The common symptoms in cancer include pain, fatigue, depression and cognitive dysfunction. The available treatment options for symptom management are limited. Methylphenidate, a psychostimulant, may be of benefit for these patients. In this report, we review the use of methylphenidate for symptoms control in cancer patients. Method: Electronic literature search on PubMed was conducted using the following keywords: methylphenidate, cancer, carcinoma, oncology, oncological and tumour. We identified forty two relevant studies and publications on the use of methylphenidate in cancer patients to be included in this review. Results: Methylphenidate was found to have some evidence in reducing opioid-induced sedation, improving cognitive symptoms and reduction of fatigue in cancer patients. Nevertheless, the results were inconsistent due to variations in the study populations, study design and outcome measures, among others. There was minimal evidence on its use in treating depression. Otherwise, methylphenidate was generally well-tolerated by patients. Conclusion: This review potentially supports the use of methylphenidate for opioidinduced sedation, cognitive decline and fatigue in cancer patients. Further placebo-controlled trials would help in strengthening the evidence for this treatment.
\end{abstract}

Keyword: Methylphenidate; Cancer; Cognition; Depression; Fatigue; Pain 